\title{
Avaliação histológica de pólipos endometriais em mulheres após a menopausa e correlação com 0 risco de malignização
}

\author{
Histopathologic features of endometrial polyps during menopause and correlation \\ with the risk for concomitant pathology
}

Adriana Bittencourt Campaner ${ }^{1}$, Soraia de Carvalho ${ }^{2}$, Sonia Maria Rolim Rosa Lima ${ }^{3}$, Roberto Euzébio dos Santos ${ }^{3}$, Maria Antonieta Longo Galvão ${ }^{4}$, Paulo Ayrosa Galvão Ribeiro ${ }^{5}$, Tsutomu Aoki ${ }^{6}$

\section{RESUMO}

Objetivos: caracterizar histologicamente pólipos endometriais em mulheres após a menopausa e avaliar o risco de lesões hiperplásicas e neoplásicas neles contidos. Métodos: análise retrospectiva de 82 casos de mulheres portadoras de histopatologia confirmatória de pólipo endometrial, submetidas à polipectomia histeroscópica devido ao diagnóstico de espessamento endometrial à ultra-sonografia transvaginal. Todos os prontuários pertinentes foram revisados para obtenção da história clínica, antecedentes, dados referentes ao exame histeroscópico e resultado final da histopatologia. Resultados: das 82 mulheres avaliadas, 10,9\% faziam uso de algum tipo de terapia hormonal e 28 (34,1\%) queixavam-se de sangramento por via vaginal. Pólipo solitário foi encontrado em 56 mulheres $(68,3 \%)$, em 19 casos $(23,2 \%)$ foram encontrados dois pólipos e em 7 casos $(3,6 \%)$, três ou mais pólipos. A avaliação histológica definitiva evidenciou pólipo benigno em 63 mulheres (76,8\%), pólipos com hiperplasia em 17 casos (20,8\%), sendo dez com hiperplasia simples sem atipias (12,2\%) e sete com hiperplasia complexa sem atipias $(8,6 \%)$. Em dois pólipos foi encontrada neoplasia associada $(2,4 \%)$. Para a análise estatística foi empregado o teste do $\chi^{2}$, corrigido por Yates. Correlacionou-se a histologia dos pólipos com a presença ou não de sangramento genital $(p=0,0056)$, o número de pólipos encontrados por mulher $(p=0,921)$, e o tempo decorrido após a menopausa $(p=0,720)$. Conclusões: pólipos endometriais são entidades freqüentemente encontradas após a menopausa, associando-se com baixa freqüência a hiperplasias e carcinomas endometriais e apenas o estudo histológico de todo espécime nos permite afastar malignidade.

PALAVRAS-CHAVE: Pólipos; Endométrio, patologia; Menopausa; Neoplasias do endométrio; Hiperplasia endometrial

\section{ABSTRACT}

Purpose: to characterize postmenopausal endometrial polyps and to determine risk for concomitant premalignant and malignant pathology. Methods: a retrospective study including 82 postmenopausal women with a histological diagnosis of endometrial polyps who underwent hysteroscopic polypectomy, after a diagnosis of endometrial thickening made by transvaginal ultrasound, was performed. Medical reports provided clinical and gynecological history, data related to the operative hysteroscopy and definitive histological findings. Results: among the 82 patients who underwent hysteroscopic polypectomy, $10.9 \%$ were receiving some type of hormonal therapy. Twenty-eight women (34.1\%) reported abnormal vaginal bleeding. Single polyp was encountered in 56 women (68.3\%), two polyps were found in 19 cases $(23.2 \%)$ and in 7 cases (3.6\%), three or more polyps were found. The definitive histopathologic analysis revealed $63(76.8 \%)$ benign polyps, 17 (20.8\%) hyperplastic polyps (10 cases $-12.2 \%$ - of simple endometrial hyperplasia without cytologic atypia and 7 cases $8.6 \%$ - of complex endometrial hyperplasia without cytologic atypia). Two polyps $(2.4 \%)$ were diagnosed as harboring neoplasia. For the statistical analysis we employed $\chi^{2}$ test improved by Yates. The authors correlated the polyps' histology

Trabalho realizado no Departamento de Obstetrícia e Ginecologia Irmandade da Santa Casa de Misericórdia de São Paulo - São Paulo (SP) - Brasil.

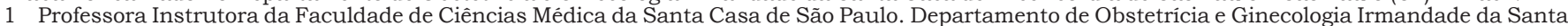
Casa de Misericórdia de São Paulo - São Paulo (SP) - Brasil.

2 Médica Assistente do Departamento de Obstetrícia e Ginecologia Irmandade da Santa Casa de Misericórdia de São Paulo - São Paulo (SP) - Brasil.

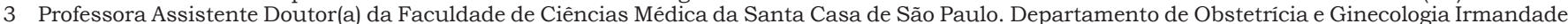
da Santa Casa de Misericórdia de São Paulo - São Paulo (SP) - Brasil.

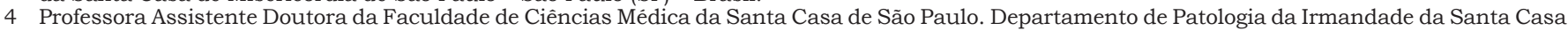
de Misericórdia de São Paulo - São Paulo (SP) - Brasil.

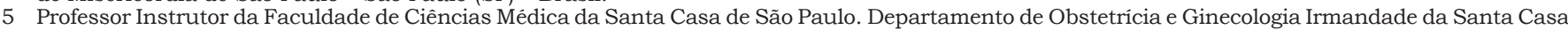
de Misericórdia de São Paulo - São Paulo (SP) - Brasil.

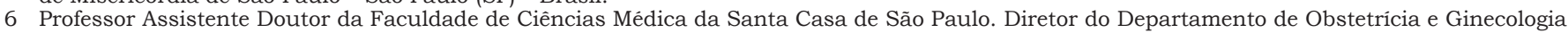
Irmandade da Santa Casa de Misericórdia de São Paulo - São Paulo (SP) - Brasil.

Correspondência: Adriana Bittencourt Campaner

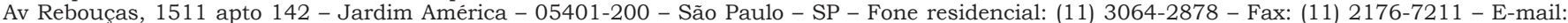
abcampaner@terra.com.br

Recebido em: 4/1/2006 Aceito com modificações em: 26/1/2006

RevBras Ginecol Obstet. 2006; 28(1): 18-23 
with the occurrence of abnormal vaginal bleeding $(\mathrm{p}=0.0056)$, number of endometrial polyps $(\mathrm{p}=0.921)$ and time after menopause $(p=0.720)$. Conclusions: endometrial polyps are commonly found entities in postmenopausal women, related with low frequency to endometrial hyperplasia or carcinomas and only histological evaluation seems to allow the exclusion of premalignant and malignant pathology.

KEYWORDS: Polyps; Endometrium, pathology; Menopause; Endometrial neoplasms; Endometrial hyperplasia

\section{Introdução}

A prevalência dos pólipos endometriais na população geral varia de 9 a $25 \%^{1-6}$, sendo os mesmos encontrados em cerca de $10 \%$ dos úteros examinados em autópsias ${ }^{1}$. Incidem preferentemente em multíparas, com maior prevalência na perimenopausa ao redor dos 40 e 50 anos, aumentando sua a prevalência, progressivamente com a idade. São raros antes da menarca, sendo relativamente freqüentes após a menopausa ${ }^{1-6}$.

Estas lesões são consideradas proliferações focais do endométrio que formam massas circunscritas, fazem protrusão para a cavidade endometrial. O tumor pode ser séssil ou pediculado e de formato ovóide ou alongado. Apresentam dimensões variáveis, sendo os mais comuns entre 2 e 4 $\mathrm{cm}$, com possibilidade de exteriorização através do óstio cervical. São geralmente solitários, porém são múltiplos em aproximadamente um quarto dos casos e associados a miomas em 40\%. Originamse em qualquer ponto da cavidade uterina, mas a maioria se localiza na região fúndica que é seguida das regiões cornuais ${ }^{1,3,4,7}$.

Microscopicamente os pólipos endometriais podem ser reconhecidos por sua projeção acima da superficie endometrial, sendo composto por glândulas, estroma e vasos sangüineos. Morfologicamente são descritos diferentes tipos de pólipos endometriais, não existindo, no entanto, consenso quanto à classificação destas estruturas, que ora segue o padrão histológico do epitélio de revestimento ora do componente estromal, ora da localização do pólipo. No entanto, vale lembrar que esta classificação tem pouco significado clínico ${ }^{1,4-5}$.

O sintoma mais comum entre as mulheres portadoras de pólipos endometriais é o sangramento uterino anormal, de intensidade variável, bem como o sangramento após a menopausa. Em raras ocasiões estas lesões podem sofrer alterações isquêmicas pela eventual diminuição do suprimento sanguíneo, evoluindo com conseqüente corrimento de odor fétido, às vezes sanguinolento e até mesmo purulento. Estas estruturas podem ser causa de dor em cólica, bem como de infertilidade por obstruírem os óstios tubários e dificultarem a implantação embrionária. No entanto, as mulheres também podem ser assintomáticas e a doença representar achado de exame ultrasonográfico (USG) ${ }^{2,7-10}$. No menacme as portadoras destas lesões apresentam maior tendência à sintomatologia das menopausadas, principalmente em relação à presença de sangramento uterino anormal ${ }^{4,6}$.

Os pólipos endometriais podem ser diagnosticados pela propedêutica subsidiária por métodos de imagem e métodos invasivos. Estes incluem a ultra-sonografia, histerossonografia, histerossalpingografia, biópsia endometrial, curetagem uterina e principalmente a histeroscopia.

Ecograficamente os pólipos endometriais caracterizam-se por espessamentos endometriais focais com aspecto hiperecogênico, sempre em continuidade com a camada basal do endométrio. No entanto, podem também se apresentar com elevada freqüência sob a forma de espessamento endometrial inespecífico e heterogêneo (contendo ou não áreas císticas), visto que estes tumores sofrem achatamento devido à compressão exercida pelas paredes do útero. Entretanto, estas lesões representam altos indices de falsos negativos quando da utilização desta técnica propedêutica ${ }^{2,5}$.

A utilização rotineira da USG transvaginal em mulheres assintomáticas no menacme e também após a menopausa tem resultado em diagnóstico cada vez mais freqüente de pólipos endometriais, os quais, antigamente, não eram descobertos $^{2,4,6,8,11}$.

A biópsia do endométrio e a curetagem uterina foram consideradas durante longo tempo métodos invasivos de excelência utilizados na avaliação da cavidade uterina. Tem-se comprovado, entretanto, que a realização destes procedimentos pode falhar na obtenção de amostras da região afetada, principalmente em casos de pólipos endometriais, o que é perfeitamente justificado pela característica fugidia destas lesões. Estas técnicas também não fornecem informações em relação à localização, tamanho e número destas lesões, sendo que os pólipos podem não ser removidos em sua totalidade ${ }^{7,9,12,13}$.

Por vezes o espécime obtido por meio da curetagem uterina apresenta-se sob a forma de fragmentos, tornando dificil o diagnóstico definitivo de pólipo, visto que o tecido encontrado pode 
assemelhar-se ao endométrio subjacente. Na pósmenopausa, a maioria dos pólipos apresenta transformação fibrosa do estroma, fator este responsável pela remoção inadequada destas lesões pela curetagem neste período de vida ${ }^{3}$.

A histeroscopia diagnóstica, por sua vez, provê visualização direta da cavidade endometrial e das lesões polipóides em seu interior. Nos permite definir o tamanho, a localização, a quantidade e o aspecto destas lesões, além de admitir biópsia dirigida às áreas suspeitas ${ }^{7,8}$.

No entanto, este importante método propedêutico pode falhar no diagnóstico diferencial visual de lesões benignas em relação às prémalignas e malignas ${ }^{6,14}$. Alguns estudos publicados demonstraram casos em que lesões confirmadas histologicamente como hiperplasias e carcinomas endometriais não haviam sido reconhecidas visualmente à histeroscopia. Estes autores recomendam, portanto, a avaliação histológica de toda a lesão polipóide para exclusão de malignidade, embora estas lesões possam apresentar aparência histeroscópica característica ${ }^{9,15}$.

Quanto a sua patogênese ainda há duvidas. Acredita-se que seu desenvolvimento esteja relacionado na maioria das vezes a estímulos hormonais, como pode ser evidenciado em casos de anormalidades endometriais em mulheres tratadas com tamoxifeno por neoplasia de mama ou em casos de utilização prolongada de estrogênios isolados. Estas lesões também ocorrem com mais freqüência em mulheres anovuladoras crônicas e inférteis ${ }^{3,16-18}$.

Sugere-se que os mesmos teriam sua origem em pequeno adenoma situado na camada basal do endométrio, apresentando assim padrão basal-símile, porém refratário às influências hormonais, mesmo em ciclo bifásico ${ }^{5}$. No menacme sua ocorrência e desenvolvimento poderiam resultar de decréscimo no estímulo dos receptores de progesterona, principalmente em suas células estromais. Já após a menopausa, geralmente não estão associados a altas concentrações séricas de estrogênios visto que o endométrio adjacente é atrófico; acredita-se que os receptores destes dois hormônios estariam presentes em quantidades maiores no epitélio glandular dos pólipos do que no endométrio adjacente, o que não ocorre da mesma maneira em seu estroma, onde existiria menor quantidade de receptores de progesterona $^{4,6,19-21}$

Foram demonstradas alterações genéticas envolvendo principalmente o cromossomo 6 , sugerindo que as alterações cromossômicas teriam papel de destaque em sua gênese ${ }^{5,22}$. Foi também descrita a expressão aumentada da oncoproteína c-erb-2 em pólipos endometriais, postulando-se que a relação desta oncoproteína com a expressão do EGF (fator de crescimento epitelial), poderia ser o mecanismo responsável pelo seu crescimento, mesmo em baixas concentrações séricas de estrogênios ${ }^{23}$.

A incidência de malignização dos pólipos descrita na literatura é variável. Hiperplasias e até mesmo carcinoma do endométrio podem estar presentes nestas lesões. Dessa maneira nosso estudo objetivou caracterizar histologicamente pólipos endometriais em mulheres após a menopausa e avaliar o risco de lesões hiperplásicas e neoplásicas neles contidos.

\section{Métodos}

O presente estudo foi realizado retrospectivamente no Departamento de Obstetricia e Ginecologia (DOGI) da Irmandade da Santa Casa de Misericórdia de São Paulo (ISCMSP) no período de maio de 2000 a maio de 2005 . O critério para inclusão de mulheres ao estudo foi de exame histopatológico confirmatório de pólipo endometrial.

Todas as 82 mulheres incluídas ao estudo haviam sido submetidas à avaliação histeroscópica da cavidade uterina, seguida de polipectomia histeroscópica, por apresentarem eco endometrial espessado e irregular à ultra-sonografia (USG) transvaginal (eco endometrial maior que $5 \mathrm{~mm}$ ). Os prontuários foram revisados para se obter dados relacionados à sintomatologia, antecedentes pessoais, antecedentes ginecológicos e obstétricos, ao exame histeroscópico e ao resultado final da histopatologia.

Foram incluídas tanto as mulheres assintomáticas bem como aquelas com sangramento uterino, independentemente de se encontrarem ou não em uso de terapia hormonal. Todas as mulheres encontravam-se após a menopausa e como tal foram consideradas caso reportassem amenorréia de pelo menos um ano de duração, após a idade de 40 anos e com FSH $\geq 30 \mathrm{mUI} / \mathrm{mL}$.

O projeto deste trabalho foi aprovado pela Comissão Científica do Departamento de Obstetrícia e Ginecologia (DOGI) e pelo Comitê de Ética em Pesquisa da Irmandade da Santa Casa de Misericórdia de São Paulo (ISCMSP).

Os diagnósticos histopatológicos foram revisados pela mesma patologista e classificados como benignos, hiperplásicos (hiperplasia simples ou complexa, com e sem atipias) e malignos, de acordo com o seu epitélio de revestimento. 
Para as análises estatísticas foi empregado o teste do $\chi^{2}$, corrigido por Yates. Correlacionou-se sua histologia definitiva com a presença ou não de sintomatologia, isto é, sangramento genital, número de pólipos encontrados por mulher e tempo decorrido após a menopausa. Para todos os testes estatísticos considerou-se o nivel de significância de $5 \%(\mathrm{p}<0,05)$.

\section{Resultados}

A idade das pacientes variou entre 42 e 82 anos, com média de 61,2 anos e mediana de 59,5 anos. Quanto à idade da menopausa, a mesma oscilou entre 40 e 60 anos, com média de 49,9 anos e mediana de 50 anos. O tempo de após a menopausa variou de 1 a 30 anos, com média de 11,2 anos e mediana de 9 anos. Do total de mulheres avaliadas (82 casos), 9 (10,9\%) faziam uso de algum tipo de terapia hormonal (TH). Os esquemas hormonais utilizados incluiam estrogênios e progestágenos combinados contínuos ou tibolona. Vinte e oito mulheres $(34,1 \%)$ queixavam-se de sangramento por via vaginal e as restantes apresentavam-se sem esta condição.

Pólipo solitário foi encontrado em 56 das 82 pacientes $(68,3 \%)$. Em 19 casos $(23,2 \%)$ foram encontrados dois pólipos, em 3 casos $(3,6 \%)$, três pólipos, em um caso $(1,3 \%)$ foram encontrados quatro pólipos e em três $(3,6 \%)$, múltiplos pólipos.

A avaliação histológica definitiva dos espécimes obtidos por meio da histeroscopia cirúrgica evidenciou pólipo benigno em 63 mulheres $(76,8 \%)$, pólipos com hiperplasia em 17 casos $(20,8 \%)$, sendo sete com hiperplasias complexas sem atipias $(8,6 \%)$ e dez com hiperplasias simples sem atipias $(12,2 \%)$. Em duas mulheres foram encontrados pólipos neoplásicos $(2,4 \%)$ (um adenocarcinoma endometrióide e um carcinoma espinocelular) (Tabela 1).

\begin{tabular}{lcr}
\multicolumn{3}{l}{ Tabela 1 - Histologia definitiva dos espécimes obtidos por meio da histeroscopia cirúrgica. } \\
\hline Histologia & $\mathbf{n}$ & $\%$ \\
\hline Pólipos benignos & 63 & 76,8 \\
Hiperplasia simples sem atipias & 10 & 12,2 \\
Hiperplasia complexa sem atipias & 7 & 8,6 \\
Neoplasias & 2 & 2,4 \\
Total & 82 & 100,0 \\
\hline
\end{tabular}

Das 63 mulheres com pólipos benignos, em $16(25,4 \%)$ verificou-se a ocorrência de sangramento genital ao passo que a mesma sintomatologia ocorreu em $12(63,1 \%)$ daquelas 19 com pólipos com alterações histológicas. Das mulheres portadoras de pólipos neoplásicos, somente uma apresentou perda sanguínea (adenocarcinoma). Empregandose o teste do $\chi^{2}$ observamos existir diferença significante $(p=0,0056)$ entre o achado histológico de pólipos benignos e patológicos quanto à presença ou não de sangramento genital. Portanto, o sangramento ocorreu com maior freqüência entre as mulheres cujos pólipos apresentavam alterações histológicas.

De maneira similar à anterior observamos que, dentre as 28 mulheres portadoras de sangramento genital espontâneo, foram encontrados à histologia 16 pólipos sem indícios de malignidade e $12(42,8 \%)$ pólipos com lesões associadas (11 casos de hiperplasias e um de neoplasia). Em relação àquelas 54 mulheres sem esta sintomatologia, encontramos 47 pólipos benignos e sete $(12,9 \%)$ com hiperplasias ou carcinoma (1 caso). Nesta comparação, utilizando-se também o mesmo teste estatístico empregado anteriormente, observamos existir diferença significante $(\mathrm{p}=0,0056)$ quanto à presença ou não de sangramento genital e o achado histológico de pólipo benignos ou associados com outras lesões.

Correlacionando-se o número de pólipos endometriais encontrados em cada mulher com sua histologia definitiva, observamos entre as $56 \mathrm{mu}-$ lheres portadoras de pólipo solitário 11 casos de hiperplasias endometriais sem atipias, dois casos de pólipos neoplásicos e o restante de lesões benignas. Nas 19 portadoras de duas lesões polipóides, encontramos quatro casos de hiperplasias endometriais sem atipias e nenhum caso de neoplasia. Já entre as sete mulheres com três ou mais pólipos, encontramos apenas dois casos de hiperplasias endometriais sem atipias e nenhum pólipo neoplásico. Nesta avaliação observamos não existir diferença significante $(p=0,921)$ entre o número de pólipos encontrados e a presença ou não de lesões patológicas nos referidos espécimes.

Por fim correlacionamos o tempo decorrido após a menopausa com a histologia definitiva das lesões em estudo. Dividimos as mulheres em quatro diferentes grupos de acordo com o tempo decorrido após a cessação das menstruações. O primeiro grupo englobou as mulheres com até cinco anos após a menopausa, o segundo aquelas entre 6 e 10 anos, o terceiro entre 11 e 20 anos e o ultimo com mais de 20 anos. A Tabela 2 expõe a histologia das lesões polipóides relacionadas aos grupos mencionados. Nesta comparação, verificamos não existir diferença significante $(p=0,720)$ entre o tempo decorrido após a menopausa e a presença ou não de lesões pré-neoplásicas ou neoplásicas. 
Tabela 2 - Correlação entre tempo após a menopausa e histologia definitiva dos pólipos endometriais.

\begin{tabular}{lrr}
\hline $\begin{array}{l}\text { Tempo de } \\
\text { pós-menopausa (anos) }\end{array}$ & \multicolumn{1}{c}{ Histologia definitiva } \\
\hline Menor ou igual a 5 & 21 & 6 hiperplasias endometriais sem atipias \\
0 neoplasias & 15 pólipos benignos \\
$6-10$ & 25 & 5 hiperplasias endometriais sem atipias \\
0 neoplasias & 20 pólipos benignos \\
$11-20$ & 23 & 2 hiperplasias endometriais sem atipias \\
2 neoplasias & 19 pólipos benignos \\
& & 4 hiperplasias endometriais sem atipias \\
0 neoplasias & 9 pólipos benignos \\
Maior que 20 & &
\end{tabular}

\section{Discussão}

Sabe-se que casos de hiperplasias e até mesmo de carcinomas do endométrio poderiam estar presentes nos pólipos endometriais. Em nossa casuística, em $17(20,8 \%)$ de nossas 82 pacientes com lesões polipóides havia a presença de hiperplasias endometriais. Em outros dois pólipos $(2,4 \%$ casos) foram registrados um adenocarcinoma do endométrio e um carcinoma espinocelular, respectivamente.

Entretanto, a freqüência das hiperplasias e dos carcinomas associados aos pólipos endometriais varia amplamente nas diversas séries publicadas ${ }^{2,7-}$ $9,11,14,21,24,25$. Para as hiperplasias os valores observados nestes estudos vão de $3,6^{9}$ a $28,8 \%{ }^{7}$. Já para o carcinoma, os referidos valores oscilaram entre $0 \%^{2}$ a $13 \%{ }^{21}$. Entretanto devemos enfatizar que estes trabalhos diferiram em sua metodologia, bem como em sua casuística, podendo-se assim explicar as diferentes incidências apresentadas.

Embora os critérios empregados para o diagnóstico definitivo nos estudos mencionados acima variassem entre a curetagem uterina e a histeroscopia cirúrgica, este fato não deve ser considerado como fator de confusão, visto que o diagnóstico histopatológico realizado nestes trabalhos se ateve ao elemento pólipo.

Sugere-se maior risco de transformação neoplásica em pacientes na pós-menopausa em relação àquelas no menacme $e^{1,6-7,10}$. Machtinger et al. ${ }^{11}$ relataram risco 17 vezes maior de desenvolvimento de lesões malignas em pólipos em mulheres após a menopausa e demonstraram que quanto maior a idade, maior será o risco de trans- formação neoplásica. Outros investigadores também encontraram achados semelhantes quanto à maior ocorrência de pólipos com malignização em pacientes na pós-menopausa ${ }^{14,21,24-25}$. Em nosso estudo não observamos correlação entre o tempo decorrido após a menopausa e o aparecimento de alterações pré-neoplásicas talvez pelo pequeno número de neoplasias encontrado.

Os pólipos que comportam hiperplasias e carcinomas apresentam maior probabilidade de sintomatologia, isto é sangramento genital, do que os pólipos sem esta condição. Este fato pode ser verificado em nossa casuística, visto que, $25,4 \%$ das portadoras de pólipos benignos apresentaram sangramento genital comparando-se aos $63,1 \%$ daquelas com lesões hiperplásicas. O risco da presença de lesões malignas e pré-malignas em pólipos de mulheres portadoras de sangramento uterino anormal pode chegar a 13,7 em comparação com as que não apresentam esta queixa ${ }^{11}$. As lesões foram observadas em 4,3\% daquelas com sangramento contra $0,5 \%$ das assintomáticas. Em outras séries, no entanto não se observou freqüência mais elevada de hiperplasia entre os casos sintomáticos em comparação com as pacientes sem sintomas ${ }^{2}$.

Entre as pacientes menopausadas, a presença de pólipo com sangramento genital eleva o risco de associação com lesão maligna em 20 vezes ${ }^{14}$. Orvieto et al. ${ }^{2}$ relataram que as pacientes em uso de terapia hormonal apresentaram taxa significantemente maior de hiperplasias endometriais do que o grupo sem sua utilização, associação não observada para o carcinoma.

Em casos de pólipos endometriais sintomáticos, pacientes inférteis e portadoras de atipias citológicas à biópsia, não costuma haver discordância entre a maioria dos ginecologistas quanto à necessidade de terapia cirúrgica para a retirada dos mesmos. No entanto, em decorrência de solicitação rotineira de métodos diagnósticos não invasivos tais como a USG, grande número de mulheres têm sido diagnosticadas como portadoras assintomáticas, gerando-se dilema em relação à necessidade ou não de terapêutica ${ }^{5}$.

A racionalidade na retirada cirúrgica dos pólipos estaria na dependência da dificuldade no seu acompanhamento, sendo que a diferenciação entre pólipos benignos e malignos só pode ser realizada definitivamente por meio de sua exérese completa. Além do mais, a remoção destas lesões estaria relacionada à melhora dos sintomas, e a cirurgia recomendada é rápida e com baixos riscos, além de tranqüilizar a mulher e o médico $2,5,7-8,10,14$.

Atualmente, a histeroscopia cirúrgica é o método mais adequado método para o tratamento cirúrgico dos pólipos endometriais, sendo que a remoção da camada basal endometrial no sítio de 
origem do pólipo poderia prevenir sua persistência e recorrência ${ }^{3,6,7,9,11,14}$.

Dessa maneira conclui-se que os pólipos do endométrio são anormalidades freqüentemente encontradas após a menopausa, associando-se com baixa freqüência a hiperplasias e carcinomas endometriais. No entanto, apenas o estudo histológico de toda a estrutura polipóide nos permite afastar tais achados. No futuro próximo, resultados de estudos em biologia molecular nos trarão informações sobre o potencial neoplásico destas lesões através de material obtido por simples biópsia histeroscópica ambulatorial.

\section{Referências}

1. Kurman RJ, Mazur MT. Benign diseases of the endometrium. In: Kurman RJ, editor. Blaustein's pathology of the female genital tract. 4th ed. New York: Springer-Verlag; 1995. p. 367- 409.

2. Orvieto R, Bar-Hava I, Dicker D, Bar J, Ben-Rafael $Z$, Neri A. Endometrial polyps during menopause: characterization and significance. Acta Obstet Gynecol Scand. 1999;78(10):883-6.

3. Reslova T, Tosner J, Resl M, Kugler R, Vavrova I. Endometrial polyps. A clinical study of 245 cases. Arch Gynecol Obstet. 1999;262(3-4):133-9.

4. Almeida ECS, Nogueira AA, Reis FJC. Princípios para a conduta em pólipos endometriais. Femina. 2003;30(9):665-7.

5. Oliveira MAP, Melki LAH, Crispi CP, Cará PR, Oliveira HC. Pólipos endometriais: diagnóstico e tratamento. Femina. 2003;31(10):885-90.

6. Nogueira AA. Pólipos endometriais. Rev Bras Ginecol Obstet. 2005;27(5):289-92.

7. Savelli L, De Iaco P, Santini D, Rosati F, Ghi T, Pignotti $\mathrm{E}$, et al. Histopathologic features and risk factors for benignity, hyperplasia and cancer in endometrial polyps. Am J Obstet Gynecol. 2003;188(4):927-31.

8. Bakour SH, Khan KS, Gupta JK. The risk of premalignant and malignant pathology in endometrial polyps. Acta Obstet Gynecol Scand. 2000;79(4):317-20.

9. Gebauer G, Hafner A, Siebzehnrubl E, Lang N. Role of hysteroscopy in detection and extraction of endometrial polyps: results of a prospective study. Am J Obstet Gynecol. 2001;184(2):59-63.

10. Goldstein SR, Monteagudo A, Popiolek D, Mayberry P, Timor-Tritsch I. Evaluation of endometrial polyps. Am J Obstet Gynecol. 2002;186(4):669-74.

11. Machtinger R, Korach J, Padoa A, Fridman E, Zolti $M$, Segal J, et al. Transvaginal ultrasound and diagnostic hysteroscopy as a predictor of endometrial polyps: risk factors for premalignancy and malignancy. Int J Gynecol Cancer. 2005;15(2):325-8.
12. Bonavolonta G, Rossetti A, Cannella PL, Campo S, Garcea N. Curettage vs. hysteroscopic resection. Minerva Ginecol. 1994;46(1-2):1-3.

13. Ghezzi F, Beretta P, Bernasconi E, Cromi A, Di Naro E, Franchi M, et al. Role of operative hysteroscopy in the removal of endometrial polyps. J Am Assoc Gynecol Laparosc. 2002;9(3 Suppl):S69.

14. Shushan A, Revel A, Rojansky N. How often are endometrial polyps malignant? Gynecol Obstet Invest. 2004;58(4):212-5.

15. de Wit AC, Vleugels MP, de Kruif JH. Diagnostic hysteroscopy: a valuable diagnostic tool in the diagnosis of structural intra-cavital pathology and endometrial hyperplasia or carcinoma? Six years of experience with non-clinical diagnostic hysteroscopy. Eur J Obstet Gynecol Reprod Biol. 2003;110(1):79-82.

16. Schlesinger C, Kamoi S, Ascher SM, Kendell M, Lage JM, Silverberg SG. Endometrial polyps: a comparison study of patients receiving tamoxifen with two controls groups. Int J Gynecol Pathol. 1998;17(4):302-11.

17. Biron-Shental T, Tepper R, Fishman A, Shapira J, Cohen I. Recurrent endometrial polyps in postmenopausal breast cancer patients on tamoxifen. Gynecol Oncol. 2003;90(2):382-6.

18. Oguz S, Sargin A, Kelekci S, Aytan H, Tapisiz OL, Mollamahmutoglu L. The role of hormone replacement therapy in endometrial polyp formation. Maturitas. 2005;50(3):231-6.

19. Mittal K, Schwartz L, Goswami S, Demopoulos R. Estrogen and progesterone receptor expression in endometrial polyps. Int $\mathrm{J}$ Gynecol Pathol. 1996;15(4):345-8.

20. Sant'Ana de Almeida EC, Nogueira AA, Candido dos Reis FJ, Zambelli Ramalho LN, Zucoloto S. Immunohistochemical expression of estrogen and progesterone receptors in endometrial polyps and adjacent endometrium in postmenopausal women. Maturitas. 2004;49(3): 229-33.

21. Hileeto D, Fadare O, Martel M, Zheng W. Age dependent association of endometrial polyps with increased risk of cancer involvement. World J Surg Oncol. 2005;3(1):8.

22. Dal Cin P, De Wolf F, Klerckx P, Van Den Berghe H. The $6 \mathrm{p} 21$ chromosome region is nonrandomly involved in endometrial polyps. Gynecol Oncol. 1992;46(3):393-6.

23. Maia H Jr, Maltez A, Fahel PE, Coutinho EM. Histochemical detection of c-erb-2 overexpression in endometrial polyps removed by hysteroscopy. Gynaecol Endosc. 2000;9(4):253-8.

24. Ben-Arie A, Goldchmit C, Laviv Y, Levy R, Caspi B, Huszar M, et al. The malignant potential of endometrial polyps. Eur J Obstet Gynecol Reprod Biol. 2004;115(2):206-10.

25.Lavie O, Ben-Arie A, Cohen S, Kedar R, Sagie S, Sharon A, et al. The risk for carcinoma and atypical hyperplasia in endometrial polyps. Int $\mathrm{J}$ Gynecol Cancer. 2004;14 Suppl 1:139-40. 